\title{
Clinicians' views on low-lying intrauterine devices or systems
}

\author{
Ellen Golightly, ${ }^{1}$ Ailsa E Gebbie ${ }^{2}$
}

${ }^{1}$ Specialist Registrar, Department of Obstetrics and Gynaecology, Royal Infirmary of Edinburgh, Edinburgh, UK

${ }^{2}$ Consultant Gynaecologist, NHS Lothian Sexual and Reproductive Health Services, Chalmers Centre, Edinburgh, UK

\section{Correspondence to} Dr Ailsa E Gebbie, NHS Lothian Sexual and Reproductive Health Services, Chalmers Centre, 2A

Chalmers Street, Edinburgh EH3 9ES, UK; ailsa.gebbie@ nhslothian.scot.nhs.uk

Received 13 May 2013 Revised 14 October 2013 Accepted 13 November 2013 Published Online First 6 January 2014

\section{SLinked}

- http://dx.doi.org/10.1136/ jpprhc-2013-100684

\begin{abstract}
Background There is a lack of consensus and very little published guidance on the management of a low-lying or malpositioned intrauterine contraceptive device (IUD) or system (IUS).
\end{abstract}

Methods and results A short e-mail questionnaire sent to senior medical staff working in contraceptive services confirmed the variation in views and management of this clinical area. Almost all respondents would replace an IUD/US lying either totally or partially in the cervical canal. The nearer the device was to the fundus the more likely respondents were to leave it in situ and there was less concern if the device was an IUS, presumably in view of the hormonal action. In the presence of abnormal bleeding or pain, most respondents would look for other causes rather than assume that the low-lying device was to blame.

Respondents expressed uncertainty as to whether low-lying devices were more likely to fail or not and around half the respondents felt that low-lying devices could migrate upwards within the cavity. Conclusion This survey highlighted the need for accurate evidence-based guidance to assist in this area of clinical contraceptive practice.

\section{BACKGROUND}

Intrauterine devices (IUDs) or levonorgestrel (LNG)-releasing systems (IUSs) are easily identified on pelvic ultrasound examination and occasionally during such scans are found to be low-lying or malpositioned within the uterus. This presents the clinician with several questions: is the device still effective, could it be causing symptoms and, of course, what should we do about it?

There is no formal published guidance on this matter and we were interested to find out what views senior specialists held and what current practice was. We designed a short survey to explore this question, the results of which are reported here.

\section{METHODS}

A short questionnaire was designed to survey the current practice of respondents

\section{Key message points}

There is no clinical consensus in how to manage a low-lying intrauterine contraceptive device (IUD) or system (IUS).

- The majority of clinicians would replace an IUD or IUS lying partially or totally within the cervical canal.

- In general, clinicians are less concerned about a low-lying IUS than an IUD, presumably because of the local hormonal effects.

with regard to low-lying IUDs/IUSs. Using the questionnaire, an informal survey of senior medical colleagues of the authors in two major centres for contraception (Edinburgh, UK and Melbourne, Australia) was performed. Respondents were approached individually by e-mail, 10 in Edinburgh and 10 in Melbourne. The response rate was $100 \%$. The following questions were asked and the response options were Yes, No or Uncertain:

1 Would you replace a low-lying IUD found incidentally on scan if it was:

(i) Totally in the cervical canal?

(ii) Partially in the cervical canal?

(iii) More than $2 \mathrm{~cm}$ below the fundus?

(iv) $1-2 \mathrm{~cm}$ below the fundus?

2 Would you replace a low-lying IUS found incidentally on scan if it was:

(i) Totally in the cervical canal?

(ii) Partially in the cervical canal?

(iii) More than $2 \mathrm{~cm}$ below the fundus?

(iv) $1-2 \mathrm{~cm}$ below the fundus?

3 If a woman has some abnormal bleeding and/or pain and a scan identifies a lowlying IUD or IUS would you:

(i) Replace the device?

(ii) Change to another contraceptive method?

(iii) Look for other causes? 
4 Do you agree with the following statements:

(i) A higher proportion of women with a low-lying copper IUD will present with bleeding or pain compared to those with a normally situated IUD.

(ii) A higher proportion of women with a low-lying levonorgestrel-releasing (LNG) IUS will present with bleeding or pain compared to those with a normally situated LNG IUS.

(iii) Women who become pregnant with a copper IUD are more likely to have a low-lying copper IUD.

(iv) A low-lying T-shaped device can migrate upwards in the uterine cavity into a normal position.

(v) Postpartum insertion is more likely to result in a low-lying position.

\section{RESULTS}

The questionnaire responses were collated. Almost all the respondents $(90 \%)$ stated that they would replace an IUD that was lying either totally or partially in the cervical canal, but fewer would do so if the IUD was low-lying but intrauterine (Figure 1). A similar trend was seen for the IUS, although all numbers were

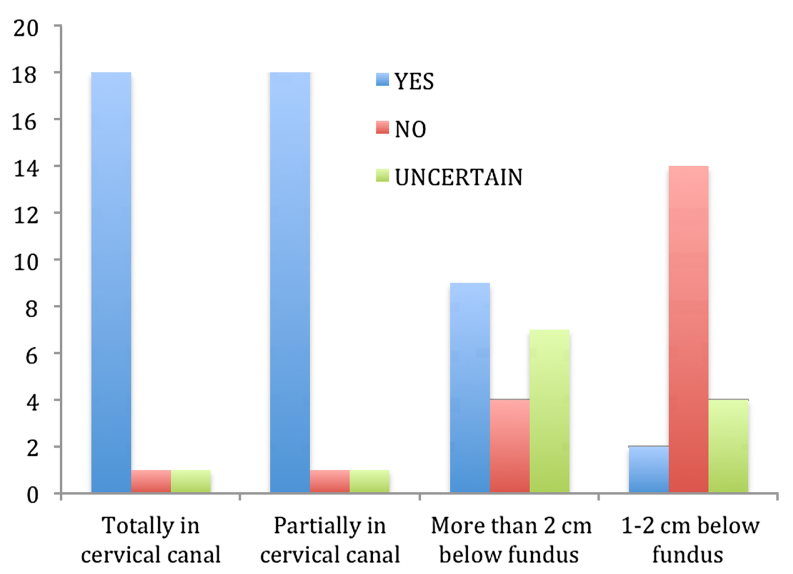

Figure 1 Clinicians' responses to the question: "Would you replace a low-lying intrauterine device found incidentally on ultrasound scan if it was ...?"

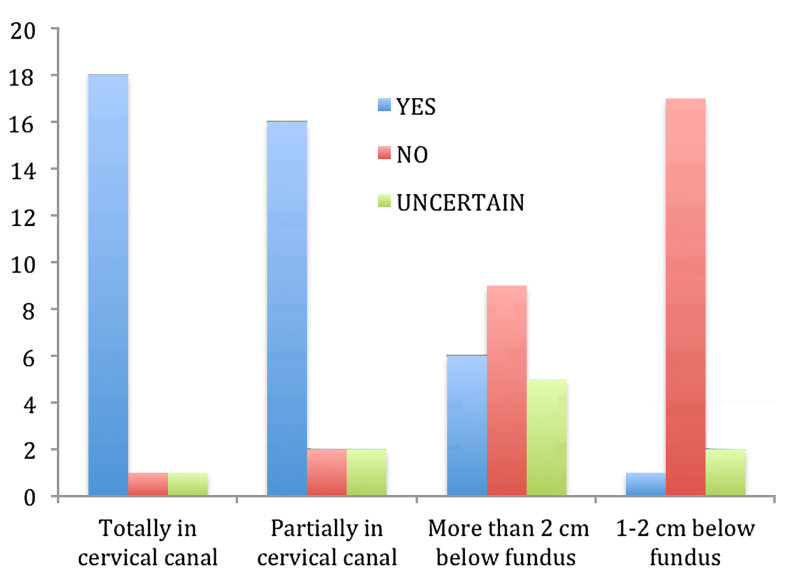

Figure 2 Clinicians' responses to the question: "Would you replace a low-lying intrauterine system found incidentally on ultrasound scan if it was ...?" lower, with only one respondent stating they would remove an IUS that was $1-2 \mathrm{~cm}$ from the fundus (Figure 2). This may suggest that the majority of respondents were concerned that a malpositioned IUD or IUS would not provide acceptable contraceptive function and that the further away it was from its optimum position, the more concerning this was. Fewer respondents were concerned when the device was an IUS, presumably because amenorrhoea is reported in some cases even when the IUS is located outside the uterus in the pelvis. ${ }^{1}$ Interestingly, $10 \%$ of respondents would not replace an IUD/IUS that was lying within the cervix. There has been a study demonstrating no difference in efficacy of a LNG-releasing intracervical device when it was placed within the cervix compared to intrauterine placement, ${ }^{2}$ and presumably respondents were reassured by this. However, the cautious approach of most respondents in wishing to replace an imperfectly sited IUD or IUS reflected the importance of effective contraception and a general 'better safe than sorry' attitude.

When it came to considering symptoms of abnormal bleeding or pain, there was mixed opinion amongst the respondents as to whether a low-lying
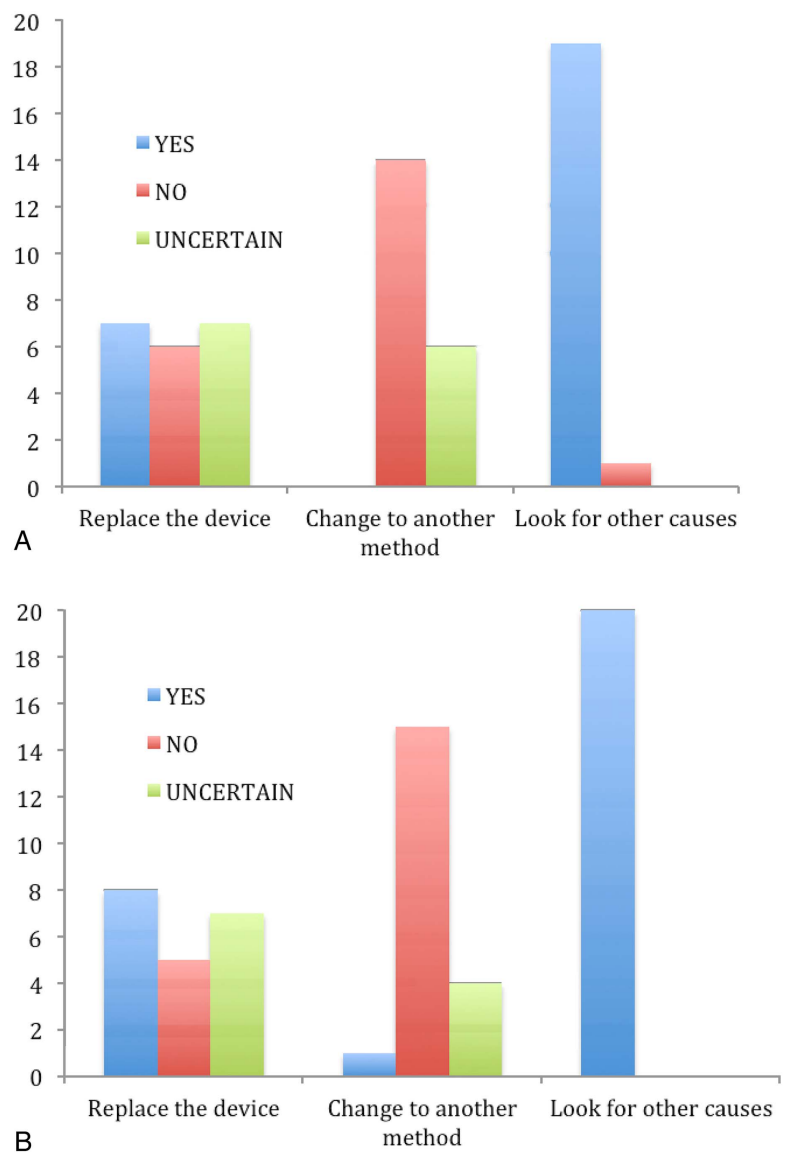

Figure 3 Clinicians' responses to the question: "If a woman has some abnormal bleeding and/or pain and a scan identifies a low-lying intrauterine device (IUD) or intrauterine system (IUS), would you in respect of the (A) IUD and (B) IUS...?". 


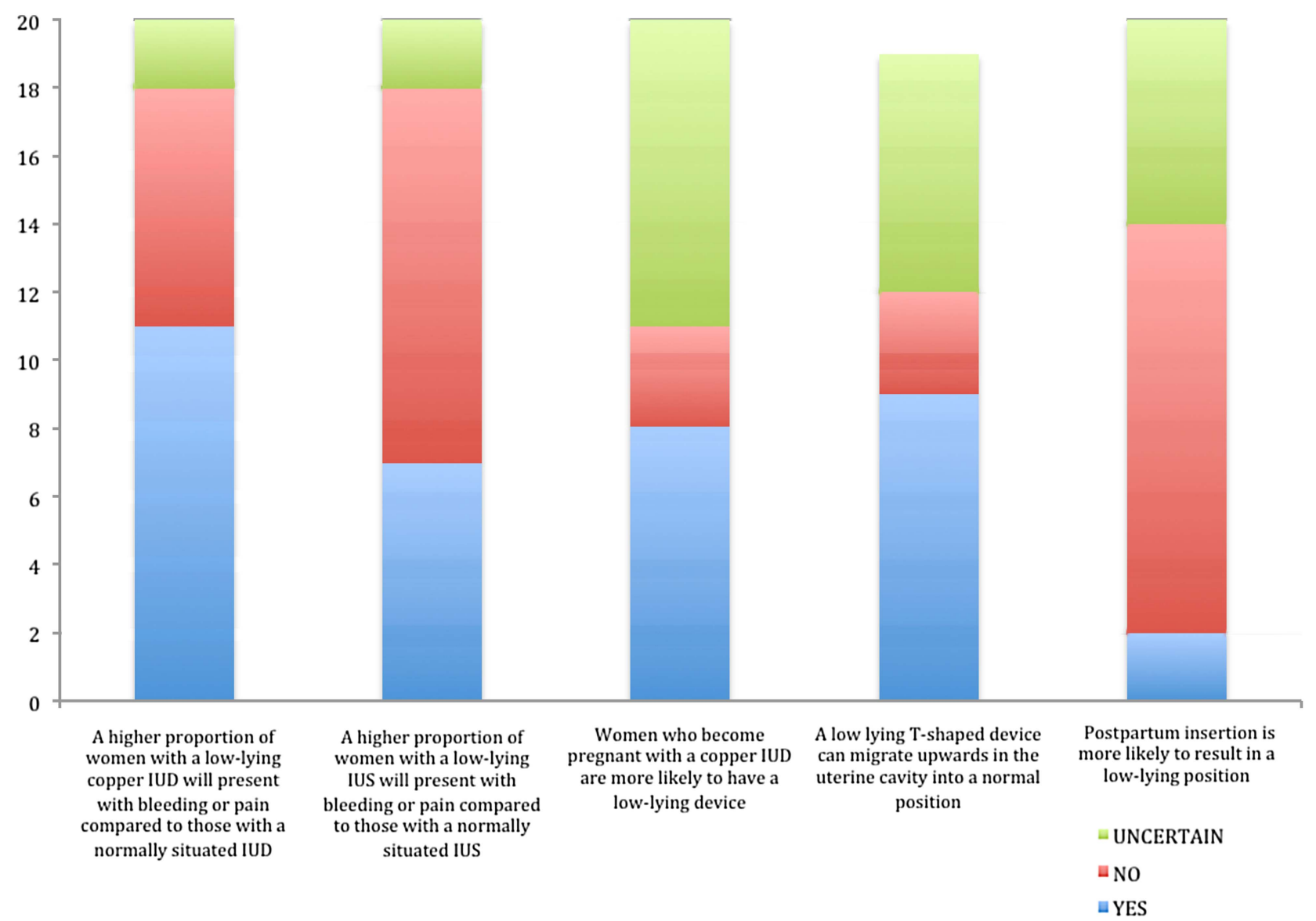

Figure 4 Clinicians' responses to the question: "Do you agree with the following statements?"

device was likely to be responsible. Almost all the respondents would look for other causes. Opinion was divided on whether removal would help to improve symptoms (Figure 3). The responses to this question reflect the general uncertainty as to the extent to which IUDs cause symptoms. Irregular bleeding and pelvic pain are not uncommon symptoms and may have many different causes. In general, the respondents were reluctant to blame the IUD or IUS without exploring all other options.

Opinion was also divided when respondents were asked if they agreed if bleeding and pain were more common in women with a low-lying IUD or IUS (Figure 4).

Almost half (45\%) of the respondents expressed uncertainty as to whether copper IUD failure was more common with low-lying IUDs, whilst $40 \%$ felt that this statement was true, and $15 \%$ that it was not true (Figure 4). This wide spread of opinion on the efficacy of a low-lying IUD reflects the paucity of published literature on this subject. Replacement of an IUD is associated with small risks of complications such as perforation or infection and can be inconvenient or uncomfortable for the patient, so ideally should not be done without good reason. Evidence-based guidance on this issue would clearly be useful.
Some $47 \%$ of respondents agreed that a low-lying T-shaped device could migrate upwards in the uterine cavity to a normal position, whilst $16 \%$ did not agree with this and 37\% were uncertain. One respondent did not answer this question (Figure 4).

Finally, most respondents did not feel that postpartum insertion was a specific risk for low-lying positioning of an IUD (Figure 4). Risk of pregnancy in the postpartum period is significant, so it is important to provide effective and convenient contraception at that time. While the majority of clinicians had overall concerns regarding the efficacy of low-lying devices, they did not feel that postpartum insertion increased the risk for devices to be low-lying and would presumably not limit their use at this time.

\section{DISCUSSION}

Our survey of senior doctors working in sexual and reproductive health demonstrated a lack of consensus on the clinical relevance of a low-lying IUD/IUS and the most appropriate way of managing that situation. Accurate evidence-based guidance would be a useful tool to guide practice and offer women safe contraception and appropriate reassurance. We have now reported on a systematic review of the published evidence, the results of which are reported separately. ${ }^{3}$ 
Our survey demonstrated that whilst opinion was mixed about the efficacy of malpositioned IUDs, most clinicians appear to replace an IUD found at least partially within the cervical canal, with confidence in the device increasing the closer an IUD is to the fundus or if the device is an IUS.

\section{Competing interests None.}

Provenance and peer review Not commissioned; externally peer reviewed.

\section{REFERENCES}

1 Bobrow C, Cooling H, Bisson D. Amenorrhoea despite displaced levonorgestrel intrauterine system. Br J Fam Plann 2000;26:105-106.

2 Pakarinen P, Luukkainen T. Five years' experience with a small intracervical/intrauterine levonorgestrel-releasing device. Contraception 2005;72:342-345.

3 Golightly E, Gebbie AE. Low-lying or malpositioned intrauterine devices and systems. J Fam Plann Reprod Health Care 2014;40:108-112.

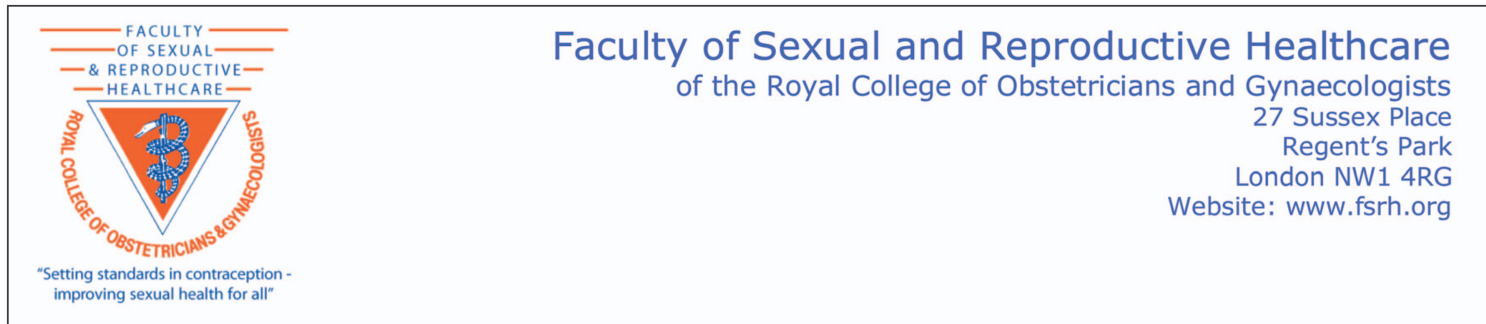

\section{VACANCY - EDITOR-IN-CHIEF}

\section{Journal of Family Planning and Reproductive Health Care}

The Journal of Family Planning and Reproductive Health Care (JFPRHC) is the quarterly, international peer-reviewed journal of the Faculty of Sexual and Reproductive Healthcare - a faculty of the Royal College of Obstetricians and Gynaecologists. The Journal, published by BMJ Publishing Group, and distributed to over 15,000 clinicians, has an impact factor of 2.100. JFPRHC aims to improve reproductive and sexual health nationally and internationally by publishing contemporary and original research covering all aspects of sexual and reproductive health relevant to clinical care, service delivery, training and education. Further information about the Journal can be found at http://jprhc.bmj.com/.

JFPRHC is seeking a new Editor-in-Chief to guide the Journal through its next phase of development, building on the success of the late Anne Szarewski and the Acting Editor-in-Chief, David Horwell.

The Editor-in-Chief needs to have an excellent understanding of the impact that a high-quality scholarly journal can have on improving knowledge and standards for healthcare professionals working in the field of sexual and reproductive healthcare. The successful candidate will engage the Journal's audiences at all levels and continue to develop its clinical content and reach. The new Editor-in Chief will lead and develop the editorial team and advance the Journal's UK and international reputation and profile, ensuring that it continues to provide engaging and informed content, and drawing on his/her extensive connections in this specialty.

Applicants need not be based in the UK; however regular travel to London for meetings of the Editorial Board and for other purposes is required. Full editorial support will be provided, as well as an annual honorarium up to the equivalent of 1.5 days a week depending on the skills and time commitment of the successful candidate.

A role description will be available on the FSRH website (www.fsrh.org) from 17 March 2014. It is envisaged that the outgoing Acting Editor-in-Chief will progressively hand over responsibility for running the Journal from 1 July 2014, with the successful candidate officially taking up the post from 1 September 2014. The term of office will be 3 years in the first instance, with an option to renew for a further 3 years on mutual agreement.

Applications should include a full CV, a letter explaining your interest in the post, your views of the strengths, weaknesses, opportunities and threats for the Journal, and an outline of what your editorial policy and vision might be. Applications should be sent to Ifountain@bmj.com by 2 May 2014. Interviews will be held in mid-May in London. 